\begin{tabular}{|c|l|}
\hline Title & Correlations in phonon-transmission spectra in periodic and quasiperiodic superlattices \\
\hline Author(s) & Tamura, S.; W atanabe, T. \\
\hline Citation & $\begin{array}{l}\text { Physical Review B, 39(8), 5349-5352 } \\
\text { https://doi.org/10.1103/PhysRevB.39.5349 }\end{array}$ \\
\hline Issue Date & 1989-03-15 \\
\hline Doc URL & http://hdl.handle.net/2115/5948 \\
\hline Rights & Copyright $\odot 1989$ A merican Physical Society \\
\hline Type & article \\
\hline File Information & PRB39-8.pdf \\
\hline
\end{tabular}

Instructions for use 


\title{
Correlations in phonon-transmission spectra in periodic and quasiperiodic superlattices
}

\author{
S. Tamura and T. Watanabe \\ Department of Engineering Science, Hokkaido University, Sapporo 060, Hokkaido, Japan
}

(Received 2 November 1988)

\begin{abstract}
We study the origin of correlations in phonon-transmission spectra in Fibonacci and periodic superlattices (SL's), which have recently been recognized by numerical calculations and then confirmed by phonon-imaging experiments. The structure factors describing the interference effects of phonons reflected from the interfaces of both periodic and Fibonacci SL's are calculated. It is found that the wave number $Q_{m, n}$ (indexed by two integers $m$ and $n$ ) for which the largest reflection occurs in a Fibonacci SL is identical to the wave number $Q_{m+n}$ for which $(m+n)$ th Bragg reflection of phonons occurs in the corresponding periodic SL. We also show more generally that major phonon-transmission dips in the Fibonacci SL occur at frequencies close to each transmission dip in the periodic SL. Two-dimensional maps for the Bragg and Bragg-like reflections of phonons in these systems indicate that the correlations are applicable, irrespective of propagation directions and modes participating in the reflection processes.
\end{abstract}

Since the discovery of quasicrystals, ${ }^{1}$ very significant advance has been made in the study of systems with quasiperiodic order. ${ }^{2}$ In particular, the spectral property of one-dimensional quasiperiodic (Fibonacci) lattices has been one of the topics studied extensively. ${ }^{3}$ The interest stems partly from the fact that the problem represents, in some sense, an intermediate case between periodic and random lattices. Undoubtedly, a major impetus toward reexamining the Fibonacci lattices was given by Merlin et $a l .{ }^{4}$ In their pioneering work they fabricated a semiconducting layered structure based on the Fibonacci sequence, known as a Fibonacci superlattice (SL). The measurements of $x$-ray scattering revealed that this artificial heterostructure indeed exhibited properties characteristic of quasiperiodic systems. ${ }^{4,5}$ Raman experiments have been performed on similar Fibonacci SL's. ${ }^{6-9}$

Recently, the phonon-transmission spectra in a Fibonacci SL have also been examined both experimental$1 y^{10}$ and theoretically. ${ }^{11}$ A striking similarity has been found between the Fibonacci and periodic SL's when the major transmission dips in the former are compared with the transmission dips in the latter. (The distribution of frequency gaps in the phonon dispersion relations also exhibits the same similarity.) It has been predicted numerically on the basis of the transfer-matrix method for both the frequency and angular dependences of the transmission. ${ }^{10,11}$ The predicted angular dependences of transmission spectra in these SL's are in reasonable agreement with the results of phonon-imaging experiment. ${ }^{10}$

The purpose of the present work is to clarify the origin of these correlations found in the phonon spectra of the periodic and Fibonacci SL's. This will be made by studying SL structure factors which describe the interference effects of phonons reflected at the interfaces of constituent layers. We shall find that the wave numbers of phonons for which the largest reflections occur in the Fibonacci SL coincide with the wave numbers satisfying the Bragg condition in the associated periodic SL. This observation leads more generally to the result that major reflections in Fibonacci SL closely correlate with Bragg reflections in the periodic SL in both their magnitudes and frequencies.

A Fibonacci SL comprises an arrangement of individual building blocks of type $A$ (each of thickness $d_{A}$ ) and type $B$ (each of thickness $d_{B}$ ) following a concatenation scheme determined by the Fibonacci sequence $G_{1}=A$, $G_{2}=A B, \quad G_{3}=A B A, \ldots, G_{r}=G_{r-1} G_{r-2} \cdot{ }^{4}$ The $r$ thgeneration Fibonacci SL $G_{r}$ consists of $F_{r}$ type- $A$ blocks and $F_{r-1}$ type-B blocks, where $\left\{F_{r}\right\}$ are the Fibonacci numbers given iteratively by $F_{r}=F_{r-1}+F_{r-2}$ for $r \geq 2$, with $F_{0}=0$ and $F_{1}=1$. In order that phonons may recognize the distinct interfaces spaced in a Fibonacci manner, each building block should be subdivided into two or more layers with different elastic properties. In this paper we assume that both type- $A$ and type- $B$ blocks consist of two layers with thickness $d_{1}^{A}$ and $d_{2}^{A}$ (with $d_{1}^{A}+d_{2}^{A}=d_{A}$ ), and $d_{1}^{B}$ and $d_{2}^{B}$ (with $d_{1}^{B}+d_{2}^{B}=d_{B}$ ). This is the case for the Fibonacci SL fabricated by Merlin et $a l .{ }^{4}$ Corresponding to the Fibonacci SL we define the associated periodic SL consisting of the sequence of alternating type- $A$ and $-B$ blocks, i.e., $A B A B A B \cdots$. Hence, the periodicity of this periodic SL is $\widetilde{d}=d_{A}+d_{B}$.

The structure factor $S_{P}$ of a regular periodic SL with periodicity $\widetilde{d}$ is given by

$$
S_{P}(Q)=\sum_{n} e^{i n Q \widetilde{d}}=\frac{2 \pi}{\widetilde{d}} \sum_{m} \delta\left(Q-Q_{m}\right),
$$

where $Q$ is the sum of the normal components of SL wave numbers for incident and reflected waves, and $Q_{m}=2 \pi m / \widetilde{d}$. The $\delta$-function peaks in Eq. (1) mean that Bragg reflection occurs for waves satisfying $Q=Q_{m}$, where $m$ stands for the order of the reflection. For a periodic SL consisting of unit cells with internal structures, the interference effects of phonons are described by the following $S_{P}^{\prime}$, which is obtainable from $S_{P}$ by taking account of extra phonon reflections properly: ${ }^{12}$ 


$$
S_{P}^{\prime}(Q)=\frac{2 \pi}{\widetilde{d}} \sum_{m} f_{P}(Q) \delta\left(Q-Q_{m}\right) \equiv \sum_{m}\left[S_{P}^{\prime}(Q)\right]_{m},
$$

where the modulation factor $f_{P}$ depends on the details of the unit-cell structure and determines the relative strength of the $\delta$-function peaks, or phonon Bragg reflections.

Here, we assume that both type- $A$ and $-B$ blocks are composed of the same kind of constituents, e.g., AlAs (first layer) and GaAs (second layer), but with different thicknesses in general (i.e., $d_{1}^{A}=d_{\mathrm{AlAs}}^{A} \neq d_{\mathrm{AlAs}}^{B}=d_{1}^{B}$ and $d_{2}^{A}=d_{\mathrm{GaAs}}^{A} \neq d_{\mathrm{GaAs}}^{B}=d_{2}^{B}$ ). In this case, $f_{P}$ can be explicitly expressed, for the normal incidence of phonons, as

$$
\begin{aligned}
& f_{P}(Q)=r_{21}\left[h_{A}(Q)+h_{B}(Q) \exp \left(i \Omega_{1}^{A}+i \Omega_{2}^{A}\right)\right], \\
& h_{I}(Q)=1-\exp \left(i \Omega_{1}^{I}\right), \quad I=A, B,
\end{aligned}
$$

where $\Omega_{n}^{A}=2 k_{n} d_{n}^{A}, \Omega_{n}^{B}=2 k_{n} d_{n}^{B}, k_{n}$ is the wave number in the $n$th layer, and $r_{21}$ is the amplitude-reflection coefficient at the interface of constituent layers (for phonons impinging on the interface from the second layer). The relation among $k_{1}, k_{2}$, and $Q$ is given by the phonon dispersion relation in this periodic system. ${ }^{12,13}$ It has been shown that $f_{P}\left(Q=Q_{m}\right)$ is proportional to the width of $m$ th frequency gap at the center or edge of the folded Brillouin zone. ${ }^{12}$.

For Fibonacci SL the structure factor $S_{F}$ is defined similarly as

$$
S_{F}(Q)=\sum_{j} \exp \left(i Q z_{j}\right),
$$

where $\left\{z_{j}\right\}$ defines the positions of the interfaces spaced by $d_{A}$ and $d_{B}$ in the Fibonacci manner. The calculation of Eq. (5) has been performed by using the projection method. $6,14,15$ The result is

$$
\begin{aligned}
S_{F}(Q)=\frac{2 \pi \tau^{2}}{d} \sum_{m, n} & \exp \left(i \phi_{m, n} / \tau^{3}\right) \\
& \times \frac{\sin \phi_{m, n}}{\phi_{m, n}} \delta\left(Q-Q_{m, n}\right),
\end{aligned}
$$

where $\tau=(\sqrt{5}+1) / 2$ is the golden mean, $d=\tau d_{A}+d_{B}$, $\phi_{m, n}=\pi \tau^{2}\left(m d_{A}-n d_{B}\right) / d$, and $Q_{m, n}=2 \pi(m+n \tau) / d$. The presence of $\delta$-function peaks in Eq. (6) indicates that Bragg-like reflections of waves also occur for $Q=Q_{m, n}$ in this quasiperiodic system, though their magnitudes are not the same but rather depend on the factor $\left(\sin \phi_{m, n}\right) / \phi_{m, n}$, i.e., the largest reflection takes place for $\phi_{m, n}=0$. To describe the interference effects of phonons, Eq. (5) should be modified again by taking account of the reflections from the boundaries of type- $A$ and $-B$ blocks as well as those from interfaces inside each block. We find that the structure factor $S_{F}^{\prime}$ relevant to the phonon reflection becomes

$$
S_{F}^{\prime}(Q)=r_{21}\left[h_{A}(Q) S_{F}^{A}(Q)+h_{B}(Q) S_{F}^{B}(Q)\right],
$$

where

$$
S_{F}^{I}(Q)=\sum_{\{j\}_{I}} \exp \left(i Q z_{j}\right), \quad I=A, B
$$

In Eq. (8) $\{j\}_{A}\left(\{j\}_{B}\right)$ are the indices indicating the location of interfaces at the beginning of type- $A$ (type- $B$ ) blocks in the Fibonacci sequence. The evaluation of $S_{F}^{A}$ and $S_{F}^{B}$ was made by Dharma-wardana et al. employing the inflation transformations. ${ }^{6}$ With the use of their results we have

$$
\begin{aligned}
S_{F}^{\prime}(Q) & =\frac{2 \pi}{d} \sum_{m, n} f_{m, n}^{F}(Q) \delta\left(Q-Q_{m, n}\right) \\
& \equiv \sum_{m, n}\left[S_{F}^{\prime}(Q)\right]_{m, n},
\end{aligned}
$$

where

$$
\begin{aligned}
f_{m, n}^{F}(Q)=r_{21}[ & h_{A}(Q) \tau \exp \left[i \phi_{m, n} \frac{1-\tau}{\tau^{3}}\right] \\
& \times \frac{\sin \left(\phi_{m, n} / \tau\right)}{\phi_{m, n} / \tau} \\
& +h_{B}(Q) \exp \left[i \phi_{m, n} \frac{1+\tau^{2}}{\tau^{3}}\right] \\
& \left.\times \frac{\sin \left(\phi_{m, n} / \tau^{2}\right)}{\phi_{m, n} / \tau^{2}}\right] .
\end{aligned}
$$

Thus, the major reflections of phonons should still occur for those $Q=Q_{m, n}$ for which $\phi_{m, n}$ is close to zero, or for integers $m$ and $n$ satisfying $m / n \cong d_{B} / d_{A}{ }^{16}$ Specifically, assuming that $d_{B} / d_{A}$ is rational, we find $Q_{M, N}=Q_{M+N}=2 \pi N / d_{A}$ for a set of integers $(M, N)$ exactly satisfying $\phi_{M, N}=0$ or $M / N=d_{B} / d_{A}$. Hence, the peak indexed by $(M, N)$ occurs at the same wave number for which $(M+N)$ th Bragg peak occurs in the periodic SL. More explicitly, the contributions of terms with this set of indices to the structure factors of the periodic and Fibonacci SL's are given by

$$
\begin{aligned}
{\left[S_{P}^{\prime}(Q)\right]_{M+N}=} & \frac{2 \pi}{\widetilde{d}} r_{21}\left[h_{A}(Q)+h_{B}(Q)\right] \\
& \times \delta\left(Q-2 \pi N / d_{A}\right), \\
{\left[S_{F}^{\prime}(Q)\right]_{M, N}=} & \frac{2 \pi}{d} r_{21}\left[h_{A}(Q) \tau+h_{B}(Q)\right] \\
& \times \delta\left(Q-2 \pi N / d_{A}\right),
\end{aligned}
$$

where we have used the equation $\exp \left[i\left(\Omega_{1}^{A}+\Omega_{2}^{A}\right)\right]=1$, which is valid for $Q=2 \pi N / d_{A}$ and in the approximation neglecting the small acoustic mismatch between the first and second layers within the type- $A$ block. In particular, for SL's in which the thicknesses of the first layers in both type- $A$ and $-B$ blocks are equal, i.e., $d_{1}^{A}=d_{1}^{B}$ but $d_{2}^{A} \neq d_{2}^{B}$ (this is the case of the Fibonacci SL grown by Merlin et al. $\left.{ }^{4}\right), \Omega_{1}^{A}=\Omega_{1}^{B}$ or $h_{A}=h_{B}$ holds and accordingly those $\delta$-function peaks in $\left[S_{P}^{\prime}\right]_{M+N}$ and $\left[S_{F}^{\prime}\right]_{M, N}$ given by Eqs. (11) and (12) are identical up to the prefactors, independent of $Q$.

The coincidence of the spectral peaks in the magnitude and wave number found above suggests more generally that even for $m / n$ not equal but close to $d_{B} / d_{A}$ the peak in $\left[S_{F}^{\prime}\right]_{m, n}$ correlates in the magnitude and wave number 
(or frequency) with the peak in $\left[S_{P}^{\prime}\right]_{m+n}$. This can be confirmed by the equation

$$
Q_{m+n}-Q_{m, n}=\frac{\phi_{m, n}}{\pi \tau^{3}} Q_{1},
$$

where $Q_{1}$ is the magnitude of the reciprocal SL vector in the periodic system. Thus, a small $\phi_{m, n}$ implies that the Bragg-like reflection in the Fibonacci SL indexed by $(m, n)$ is a major reflection and the corresponding wave number $Q_{m, n}$ is close to $Q_{m+n}$ at which the $(m+n)$ th Bragg reflection occurs in the periodic SL. More importantly, in the vicinity of each Bragg peak of the periodic SL we can find a major Bragg-like peak of the Fibonacci SL.
To see this, we assume that $d_{B} / d_{A}<1$ and write $\phi_{m, n} / \tau$ in the form

$$
\frac{\phi_{m, n}}{\tau}=\pi \frac{m-n d_{B} / d_{A}}{1+d_{B} / d_{A} \tau} .
$$

For any positive integer $l$ we can always find a set of integers $\left(m^{\prime}, n^{\prime}\right)$ which satisfy $m^{\prime}+n^{\prime}=l$ and $\left|m^{\prime}-n^{\prime} d_{B} / d_{A}\right| \leq 1$, and hence

$$
\left|\phi_{m^{\prime}, n^{\prime}} / \tau^{2}\right|<\left|\phi_{m^{\prime}, n^{\prime}} / \tau\right| \leq \pi /\left[1+\left(d_{B} / d_{A} \tau\right)\right]
$$

and

$$
\left|Q_{l=m^{\prime}+n^{\prime}}-Q_{m^{\prime}, n^{\prime}}\right|<Q_{1} / \tau^{2}\left[1+\left(d_{B} / d_{A} \tau\right)\right]
$$

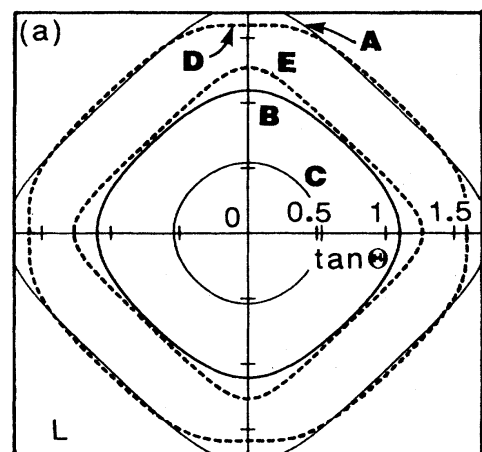

A : $L \rightarrow L(1)$

B : $L \rightarrow L$ (2)

C: $L \rightarrow L(3)$

D: L $\rightarrow$ ST (3)

E: $L \rightarrow F T$ (3)
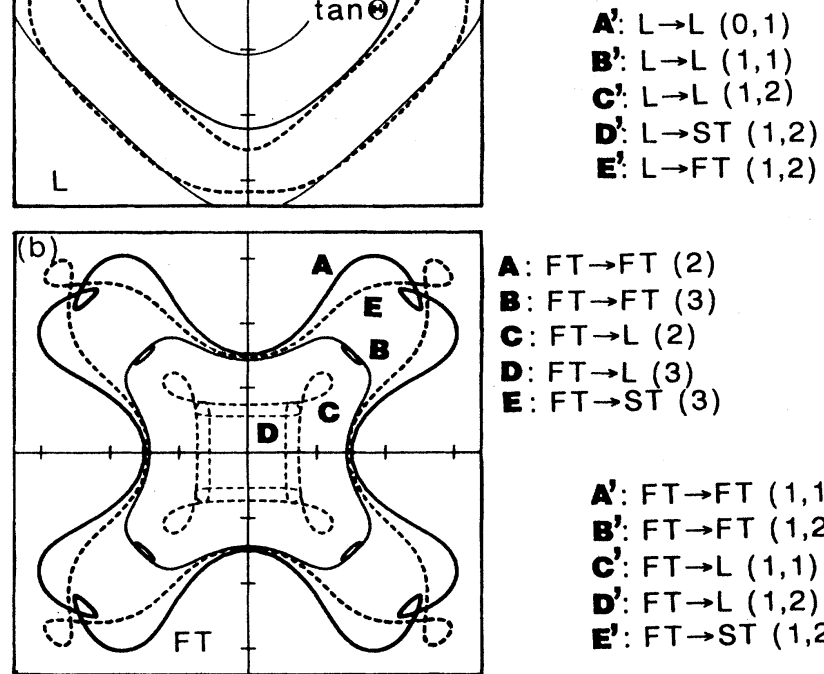

A: $F T \rightarrow F T$ (2)

$\mathbf{B}: \mathrm{FT} \rightarrow \mathrm{FT}$ (3)

C: $F T \rightarrow L$ (2)

D: $F T \rightarrow L(3)$

E: FT $\rightarrow$ ST (3)

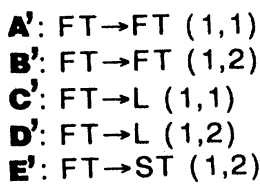

A: $F T \rightarrow F T(1,1)$

B: $F T \rightarrow F T(1,2)$

C: $F T \rightarrow L(1,1)$

$E^{\prime}:$ FT $\rightarrow$ ST $(1,2)$
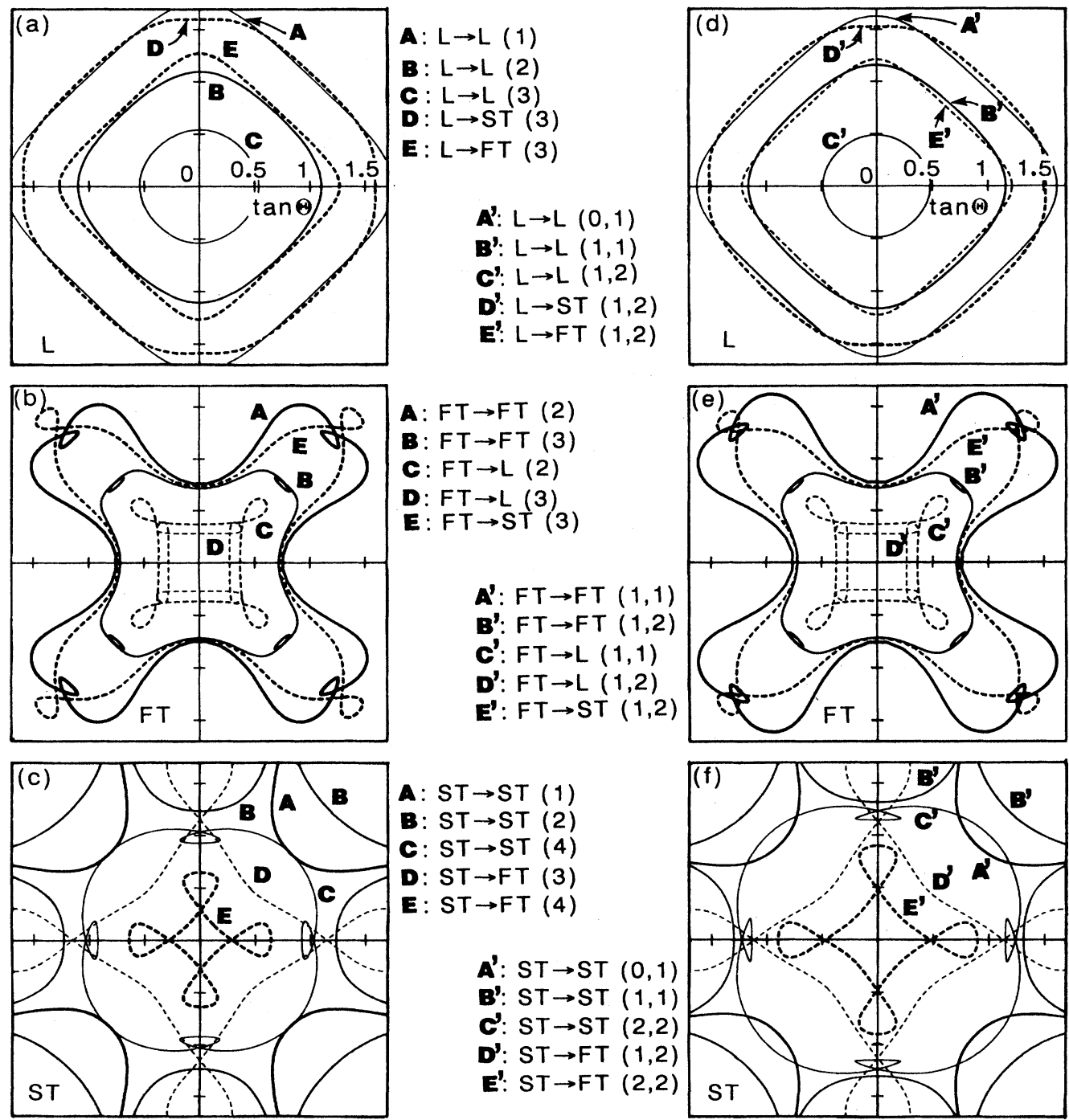

A: $\mathrm{ST} \rightarrow \mathrm{ST}$ (1)

B: $S T \rightarrow S T$ (2)

C: $S T \rightarrow S T$ (4)

D: $S T \rightarrow F T$ (3)

E: $S T \rightarrow F T$ (4)

A': ST $\rightarrow$ ST $(0,1)$
B': ST $\rightarrow$ ST $(1,1)$
C:: ST $\rightarrow$ ST $(2,2)$
D': ST $\rightarrow$ FT $(1,2)$
E: $S T \rightarrow F T(2,2)$
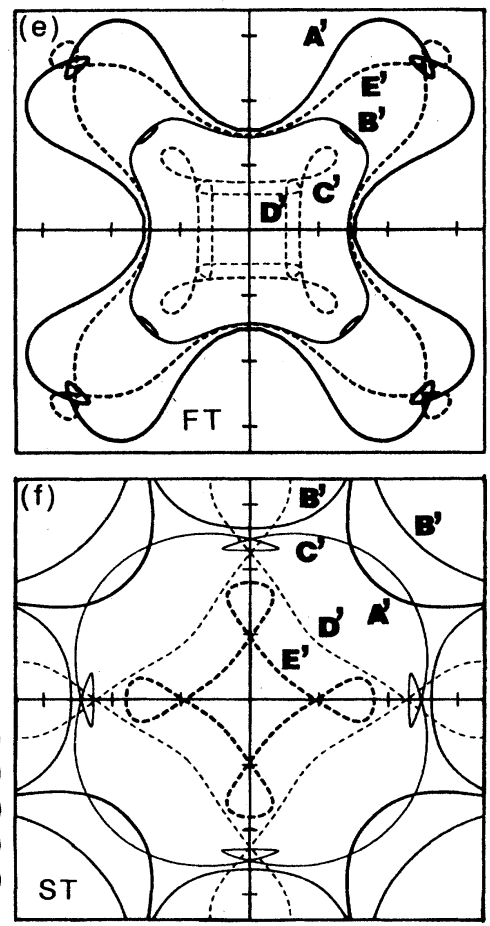

FIG. 1. 2D maps for Bragg and Bragg-like reflections in (100) AlAs/GaAs SL's; (a)-(c) for phonons in the periodic SL, and (d)-(f) for phonons in the Fibonacci SL. (FT and ST denote the fast transverse and slow transverse modes, respectively.) The thicknesses of layers in a type- $A$ block are $d_{1}^{A}=d_{\mathrm{AlAs}}^{A}=17 \AA$ and $d_{2}^{A}=d_{\mathrm{GaAs}}^{A}=42 \AA$, and those in a type- $B$ block are $d_{1}^{B}=d_{\mathrm{AlAs}}^{B}=17 \AA$ and $d_{2}^{B}=d_{\mathrm{GaAs}}^{B}=20 \AA$, such that $\left(d_{1}^{B}+d_{2}^{B}\right) /\left(d_{1}^{A}+d_{2}^{A}\right)=d_{B} / d_{A}=0.627 \cong 1 / \tau$. Phonon frequency is $850 \mathrm{GHz}$. The maps span $\pm 59.5^{\circ}$ $(\tan \Theta= \pm 1.7)$ left to right. The center of the maps is $\langle 100\rangle$ direction and the diagonals are parallel to $\{100\}$ planes. Solid and broken lines show the intramode and intermode reflections, respectively. The mode pairs participating in the reflection processes are given in the center column together with the orders of reflection [a pair of indices $(m, n)$ for Fibonacci SL] in the parentheses. 
(Ref. 17). These inequalities together with Eqs. (2), (9), and (10) mean that for the lth-order Bragg reflection of phonons in the periodic SL we can find with large probability a correlated major Bragg-like reflection in the Fibonacci SL with indices $\left(m^{\prime}, n^{\prime}\right)$ satisfying $m^{\prime}+n^{\prime}=l$. All these observations establish the close correlation in phonon-transmission dips which was previously recognized by numerical calculations ${ }^{11}$ and confirmed by phonon imaging. ${ }^{10}$

Noting that $Q$ refers to the component of phonon wave vectors parallel to the growth direction of SL's, we can expect the correlation in transmission spectra also for phonons propagating obliquely relative to the interfaces. An interesting feature of the oblique phonon propagation in SL's is the presence of intermode reflection in addition to ordinary intramode reflection. ${ }^{18,19}$ The intermode reflections, for instance, from the longitudinal (L) to transverse $(\mathrm{T})$ phonons, and vice versa, are predicted by putting $Q=q_{\mathrm{L}}+q_{\mathrm{T}}$, where $q_{\mathrm{L}}$ and $q_{\mathrm{T}}$ denote the normal components of the incident (reflected) L and reflected (incident) $\mathrm{T}$ phonons, respectively. The simplest way to observe such a spatial correlation is to plot two-dimensional (2D) maps of propagation directions satisfying $Q=Q_{m+n}$ and $Q=Q_{m, n}$ for a fixed frequency. In Fig. 1 we have shown such 2D maps for phonons in (100) AlAs/GaAs SL's on a GaAs substrate. Frequency assumed is 850 $\mathrm{GHz}$, i.e., the cut-on frequency of a $\mathrm{Pb}-\mathrm{Bi}$ tunnel junction, ${ }^{19}$ and lines indicate the directions in the GaAs substrate (in the real space) at which the Bragg or Bragg-like reflection conditions are satisfied. The modes and order of these reflections are also indicated. It should be remarked that for the Fibonacci SL we have plotted the directions of only major reflections. (The current frequency and angular resolutions of phonon detectors would make it possible to observe only major reflections yielding large transmission dips.) It is now well understood that even a small amount of elastic anisotropy of constituent layers leads to marked anisotropy in Bragg conditions, especially for $\mathrm{T}$ phonons as shown in Fig. $1 .{ }^{19}$

Comparing the 2D maps for the periodic and quasiperiodic SL's, the similarity in their spatial distributions is readily recognized. Specifically, the correspondence between the modes participating in the reflection processes and that of the orders of reflection-i.e., $(m, n)$ for the Fibonacci and $(m+n)$ for the periodic SL's - is quite remarkable. Similar results are also obtained for (111) AlAs/GaAs SL's. Here, we remark that the assumed Fibonacci SL is the same as the one used in the phononimaging experiment and lines in Fig. 1(d) coincide well with the observed attenuation directions of transmitted $\mathrm{L}$ phonons. ${ }^{10}$

To conclude, we have established close correlations in transmission spectra of phonons in periodic and quasiperiodic SL's, which exist in both the frequency and angular dependences of the transmission. Especially, the spatial distribution of L-phonon stop bands in the Fibonacci SL observed experimentally in phonon images coincides well with the 2D map of major Bragg-like reflections predicted in this paper. Because these correlations are directly derived from the properties of SL structure factors, they should also be observable in the spectra of x-ray and Raman scatterings.

\section{ACKNOWLEDGMENT}

Support for this work was provided by a Grant-in-Aid for Scientific Research from the Ministry of Education, Science and Culture of Japan, No. 63550001.
${ }^{1}$ D. Schechtman, I. Blech, D. Gratias, and J. W. Cahn, Phys. Rev. Lett. 53, 1951 (1984).

${ }^{2}$ See, for example, The Physics of Quasicrystals, edited by P. J. Steinhardt and S. Ostlund (World Scientific, Singapore, 1987).

${ }^{3}$ For a recent review, see R. Merlin, IEEE J. Quantum Electron. QE-24, 1791 (1988).

${ }^{4}$ R. Merlin, K. Bajema, R. Clarke, F.-Y. Juang, and P. K. Bhattacharya, Phys. Rev. Lett. 55, 1768 (1985).

${ }^{5}$ J. Todd, R. Merlin, R. Clarke, K. M. Mohanty, and J. D. Axe, Phys. Rev. Lett. 57, 1157 (1986).

${ }^{6}$ M. W. C. Dharma-wardana, A. H. MacDonald, D. J. Lockwood, J.-M. Barbibeau, and D. C. Houghton, Phys. Rev. Lett. 58, 1761 (1987).

${ }^{7}$ M. Nakayama, H. Kato, and S. Nakashima, Phys. Rev. B 36, 3472 (1987).

${ }^{8}$ K. Bajema and R. Merlin, Phys. Rev. B 36, 4555 (1987).

${ }^{9}$ D. J. Lockwood, A. H. MacDonald, G. C. Aers, M. W. C. Dharma-wardana, R. L. S. Devine, and W. T. Moore, Phys. Rev. B 36, 9286 (1987).

${ }^{10}$ D. C. Hurley, S. Tamura, J. P. Wolfe, K. Ploog, and J. Nagle,
Phys. Rev. B 37, 8829 (1988).

${ }^{11}$ S. Tamura and J. P. Wolfe, Phys. Rev. B 36, 3491 (1987).

${ }^{12}$ S. Tamura and J. P. Wolfe, Phys. Rev. B 38, 5610 (1988).

${ }^{13}$ S. M. Rytov, Akust. Zh. 2, 71 (1956) [Sov. Phys. Acoust. 2, 68 (1956)].

${ }^{14}$ R. K. P. Zia and W. J. Dallas, J. Phys. A 18, L341 (1985).

${ }^{15}$ V. Elser, Phys. Rev. B 32, 4892 (1985).

${ }^{16}$ Actually, $\phi_{m, n}=0$ is a necessary condition for $\left|f_{m, n}^{F}\right|$ being large. Whether $\left|f_{m, n}^{F}\right|$ is really large or not depends on the magnitudes of $h_{A}$ and $h_{B}$ which also govern through Eq. (3) the strength of Bragg peaks in the periodic SL. In this paper we refer to "major reflections" by those with small $\phi_{m, n}$.

${ }^{17}$ For $d_{B} / d_{A}>1$ we can find a set of integers $\left(m^{\prime}, n^{\prime}\right)$ satisfying $\left|\phi_{m^{\prime}, n^{\prime}} / \tau^{2}\right|<\left|\phi_{m^{\prime}, n^{\prime}} / \tau\right| \leq \pi \tau /\left(1+\tau d_{A} / d_{B}\right), \quad$ and $\quad \mid Q_{l=m^{\prime}+n^{\prime}}$ $-Q_{m^{\prime}, n^{\prime}} \mid \leq Q_{1} /\left(1+\tau d_{A} / d_{B}\right)$, where $l=m^{\prime}+n^{\prime}$ is a given integer. Hence, $\phi_{m^{\prime}, n^{\prime}} / \tau$ is not necessarily small, though $Q_{l=m^{\prime}+n^{\prime}}$ and $Q_{m^{\prime}, n^{\prime}}$ are still close to each other.

${ }^{18}$ S. Tamura and J. P. Wolfe, Phys. Rev. B 35, 2528 (1987).

${ }^{19}$ S. Tamura, D. C. Hurley, and J. P. Wolfe, Phys. Rev. B 38, 1427 (1988). 